\title{
THE BASIC ANATOMY OF SCOLIOSIS
}

\author{
ROBERT ROAF, LIVERPOOL, ENGLAND
}

Professor of Orthopaedic Surgery, University of Liverpool

This paper is primarily a plea for study of the morphology of scoliotic spines-a subject which has been neglected in this country, though considerable work on it has been done on the continent of Europe.

Many surgeons and physicians still refer to the deformity of scoliosis as that of a kyphoscoliosis. If kyphosis means an increase in the posterior elements of the vertebral column relative to the anterior elements the use of the term in this context is certainly erroneous.

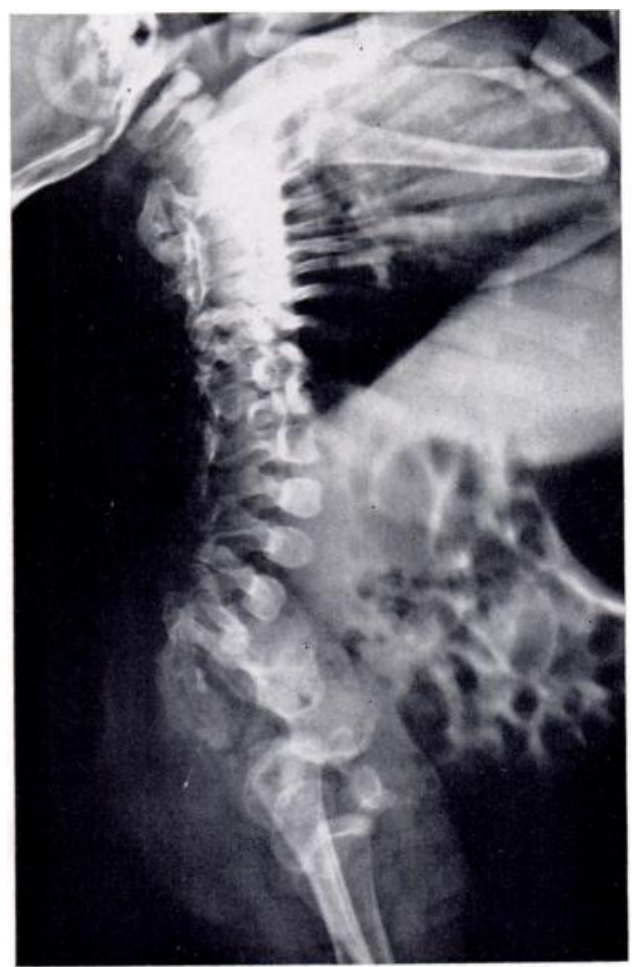

FiG. 1
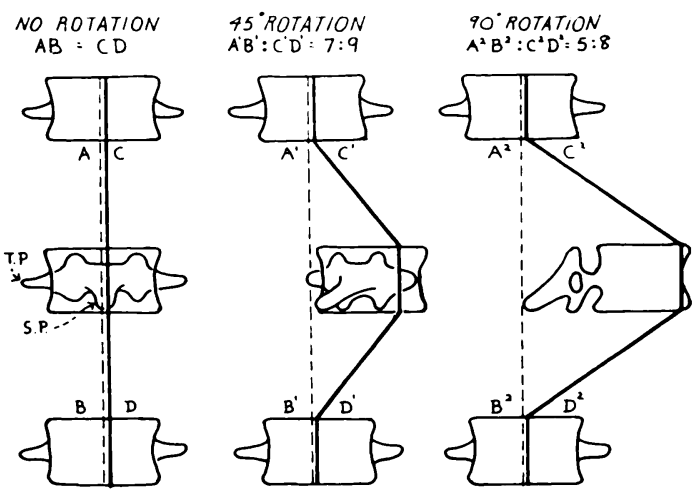

$C D, C^{\prime} D^{\prime} C^{2} D^{2}=$ Line of anterior longitudinal ligament $A B, A^{\prime} B^{\prime} \cdot A^{2} B^{2}=$ Line of interspinous ligaments

Fig. 2

FIGS. 1 AND 2

Figure 1-Total lordosis caused by either a rare congenital anomaly or complete paralysis of the abdominal muscles. Figure 2-Diagram to illustrate the mathematical relationship between relative lengthening of the anterior elements and the degree of rotation.

Observations on museum specimens and at operations, careful inspection of radiographs and examinations of necropsy material show that, in scoliotic spines, there is always a lordosis. One understands lordosis to be a lengthening of the anterior elements relative to the posterior elements.

It is possible to have a total lordosis without rotation or lateral curvature (Fig. 1), but usually the length of the sternum and tightness of the abdominal muscles prevent this. Under the restraining influence of these structures the increased length of the anterior elements can be accommodated only by lateral deviation and rotation of the vertebral bodies, and the degree of torsion varies with relative lengthening of the anterior elements (Fig. 2).

Whatever the primary cause either of the torsion or of the relative overgrowth of the anterior elements, once there is a certain amount of relative overgrowth it tends to 


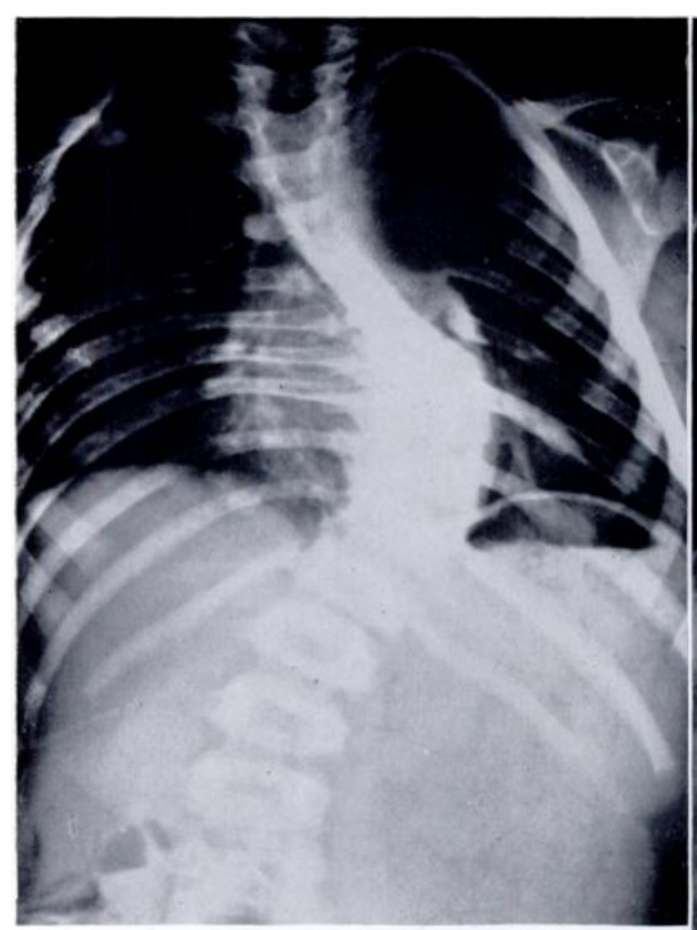

FIG. 3

Figs. 3 AND 4

Figure 3-Thoracic scoliosis before operation. Figure 4-After anterior wedge excision of the lumbar spine and application of a plaster jacket, which should increase the thoracic curve, the thoracic curve actually becomes less. This is a regular finding in young children.

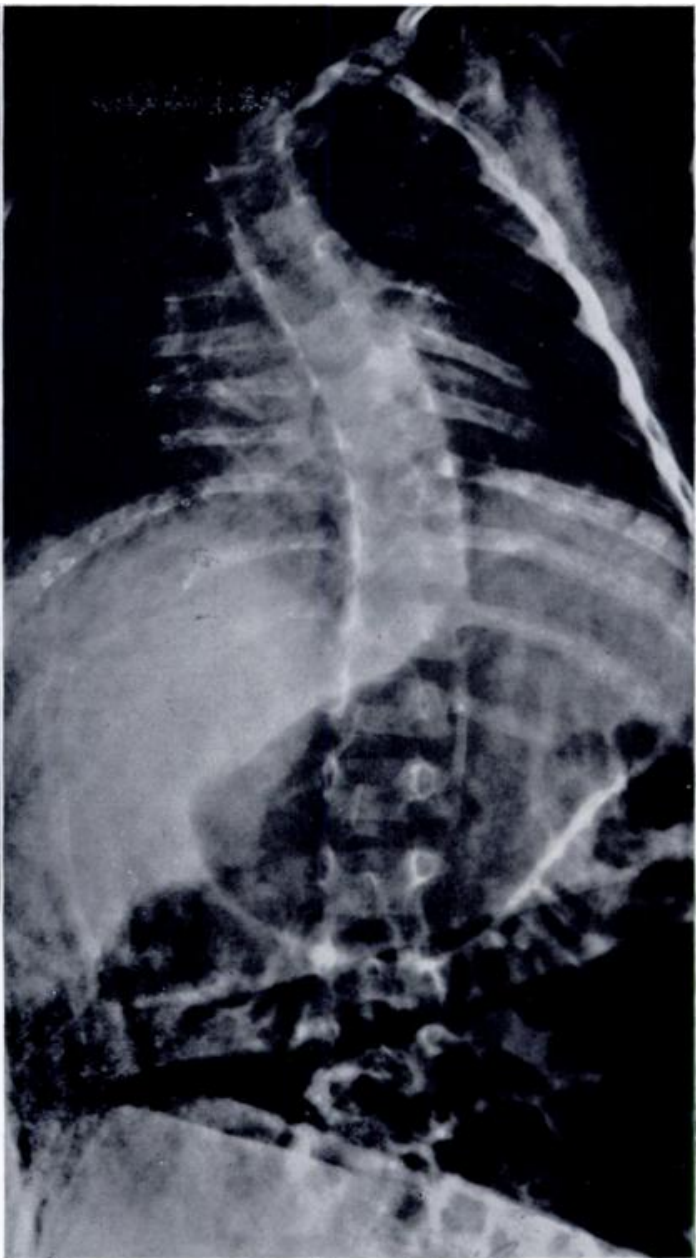

FIG. 4

perpetuate itself and the rotation and lateral flexion deformity will be corrected only if the difference in length between the anterior and posterior elements is corrected. This can be achieved by increasing the length of the posterior elements-for example by stretching. physiotherapy and braces-or by the insertion of appliances such as the Allan jack or the Harrington rod. But also it can be corrected by diminishing the length of the anterior elements either by epiphysiodesis (Roaf 1963) or wedge excision; in this connection it is possible by anterior wedge excision of the lumbar vertebral bodies, as in Chaklin's (1958) operation, to correct a thoracic curve. Diminishing the total length of the anterior elements automatically corrects the torsion and lateral flexion (Figs. 3 and 4).

It is nearly 100 years since Meyer (1866) suggested that the vertebral column may be considered as consisting of two components, namely the body series (Körperreihe), and the arch series (Bogenreihe). He pointed out that the anterior components were well adapted to withstand compression but that the posterior components were under constant elastic tension and had a great tendency to shorten. The full clinical significance of these observations has not yet been fully appreciated.

During the latter half of the nineteenth century the anatomy of scoliosis was studied and described by a number of Continental anatomists, and their work culminated in the studies of Nicolodani (1909). Modern and comprehensive studies have been recently published by Movshovich (1960, 1963). 

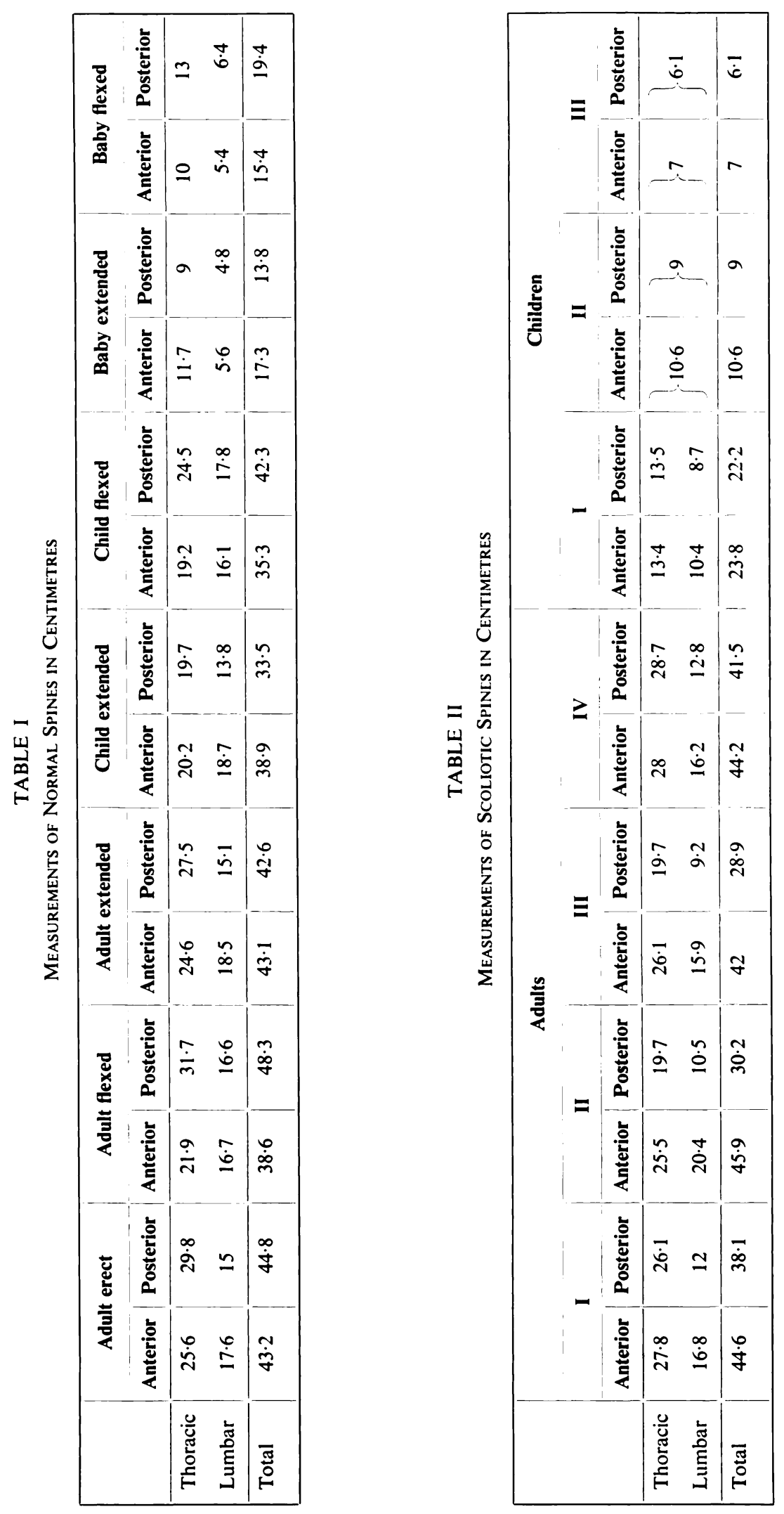

THE JOURNAL OF BONE AND JOINT SURGERY 
At first it might be thought that there was nothing more to add to these descriptions, but there is one aspect in which the earlier descriptions are inadequate. Although Nicolodani distinguished to a certain extent between the apex vertebrae and the transitional vertebrae he did not point out all the differences in the types of deformity between the apex of the curve and the transitional portion.
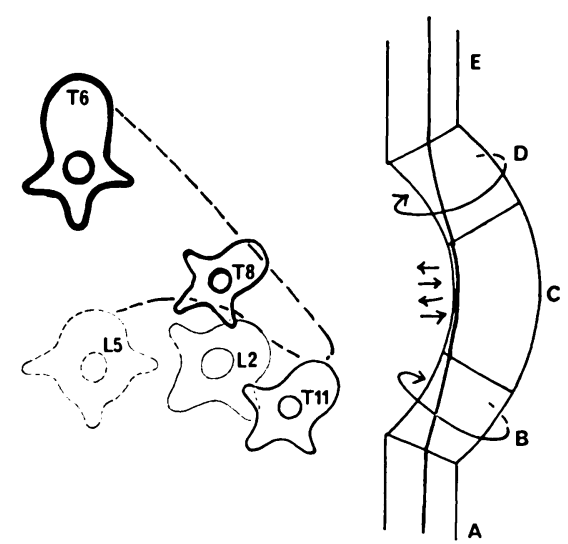

Fig. 5

The five regions of a scoliotic curve: $A$ ) pure lordosis; B) lordosis and rotation; C) lateral flexion; D) counter rotation; E) lordosis.

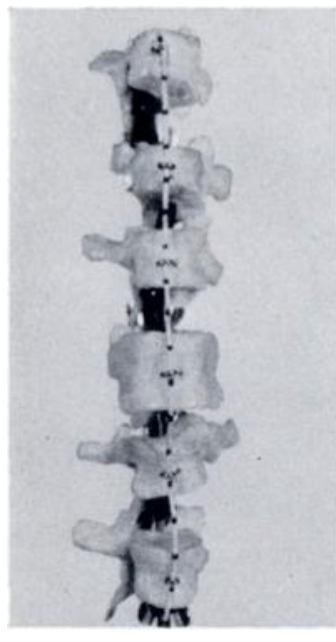

Fig. 6

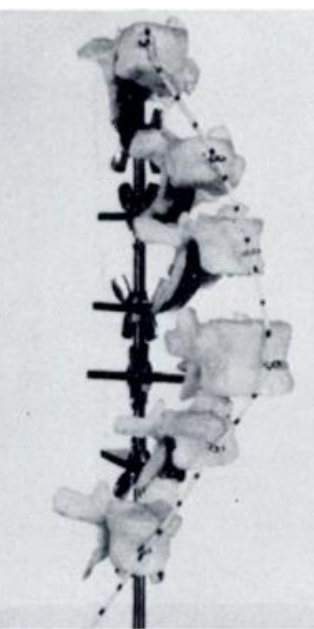

Fig. 7

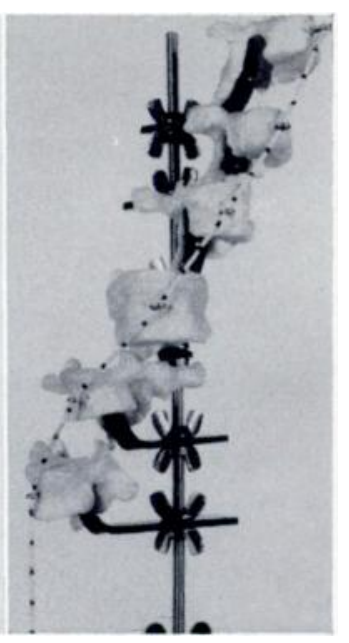

FIG. 8

With the spine straight (Fig. 6) the anterior longitudinal ligament measures 17 centimetres; with rotation (Fig. 7) it measures 23 centimetres; it is the same when the central vertebra is the neutral point (Fig. 8).

\section{MATERIAL AND FINDINGS}

The spines of four adults and of three children aged three, five and six years were available for this study. If a normal spine is measured in the upright position from the first thoracic to the fifth lumbar vertebra the sum of the lengths of all the interspinous ligaments is slightly greater than the length of the anterior longitudinal ligament over the corresponding area of the spine (Table I). In full flexion the posterior measurement is considerably longer than in full extension. By contrast, in all the scoliotic spines that $I$ have measured, the anterior 
longitudinal ligament has been longer than the sum of the corresponding interspinous ligaments (Table II). In other words, whatever the first or initiating cause of scoliosis, the result is either a relative or absolute shortening of the posterior components or relative lengthening of the anterior components; this is of considerable clinical and therapeutic significance. The scoliotic spine can be compared to the spiral staircase where the inner ends of the steps correspond to the interspinous ligaments and the outer ends to the anterior longitudinal ligament.

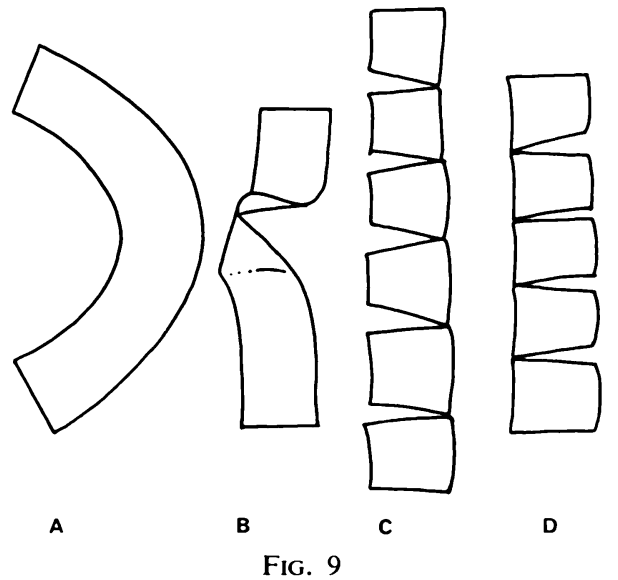

FIG. 9

A " C" shaped piece of cardboard viewed from the side (A). The long edge represents the anterior longitudinal ligament. If it is straightened, that is, the lordosis corrected by making the upper and lower ends parallel to each other. the longer - or anterior-edge bends and twists sideways (B). This could only be prevented if the discrepancies between the anterior and posterior edges are corrected either by lengthening the posterior edge $(C)$ or by shortening the anterior edge (D).

Fig. 9

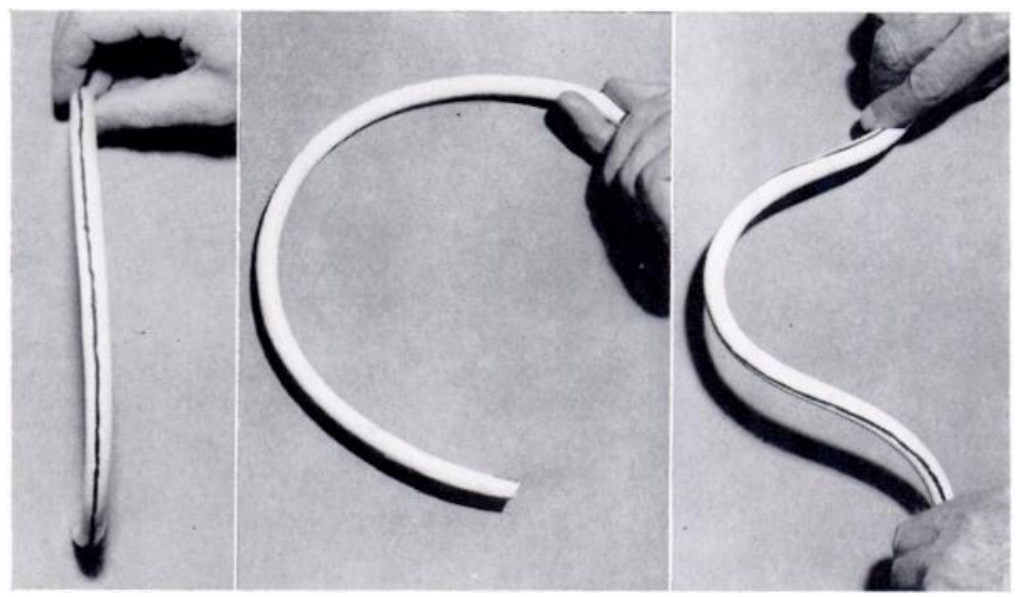

Fig. 10

Fig. 11

FIG. 12

Model to illustrate the relationship between lordosis and torsion. Figure 10-An anterior view of a piece of rubber tube in which there is a "C " shaped piece of plastic material which is capable of twisting and side bending. Figure 11-A lateral view of the same model. Figure 12-The anterior view after trying to correct the lordosis; the anterior edge (black line), representing the anterior longitudinal ligament, deviates and twists sideways.

Usually a scoliotic spine can be divided into five regions. Starting below there is, first, a total lordosis; then rotation and lordosis; next, a central part with almost true lateral flexion often with some lordosis; very occasionally in this region there is some kyphosis; above this there is counter-rotation and lordosis, and, finally, true lordosis (Fig. 5).

Simple three-dimensional geometry shows that if the vertebral bodies are rotated farther from the mid-line than the spinous processes there must be lordosis-that is, relative lengthening of the anterior longitudinal ligament (Figs. 6 to 8). In the rare condition of true kyphoscoliosis the converse holds; that is, the spinous processes are displaced more than the vertebral bodies. 


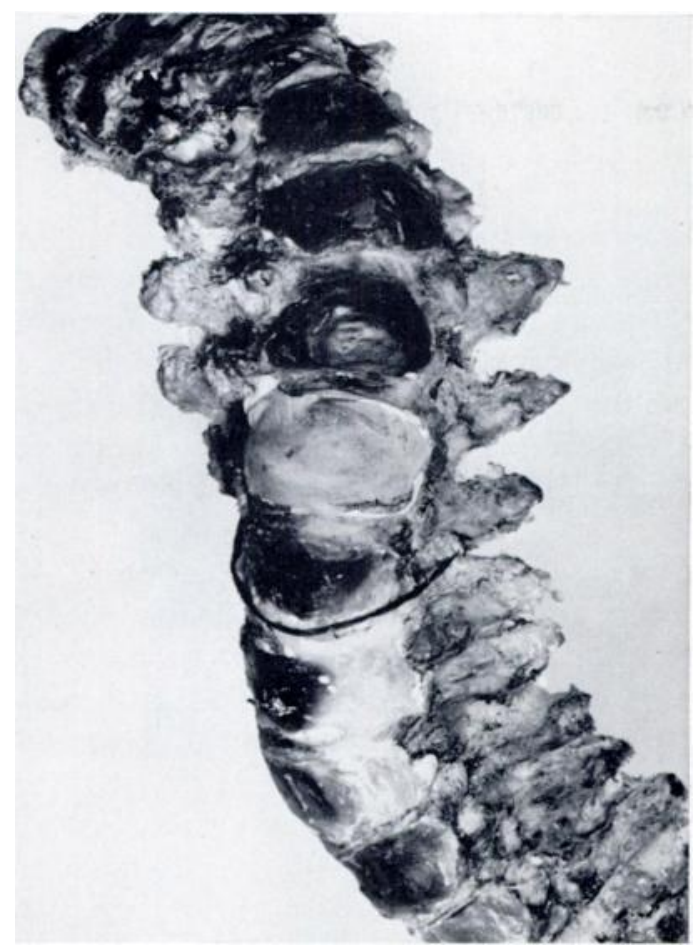

FIG. 13

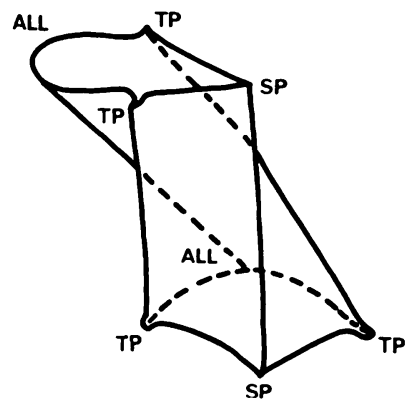

FIG. 14
FIGs. 13 AND 14

Figure 13-A photograph of the transitional part of a curve in which there is rotation and lordosis. Figure 14 is a diagram of the same part. The line SP-SP represents the line of the spinous processes. The line ALL-ALL represents the line of the anterior longitudinal ligaments. Note that line ALL-ALL is longer than SP-SP (TP : transverse process).

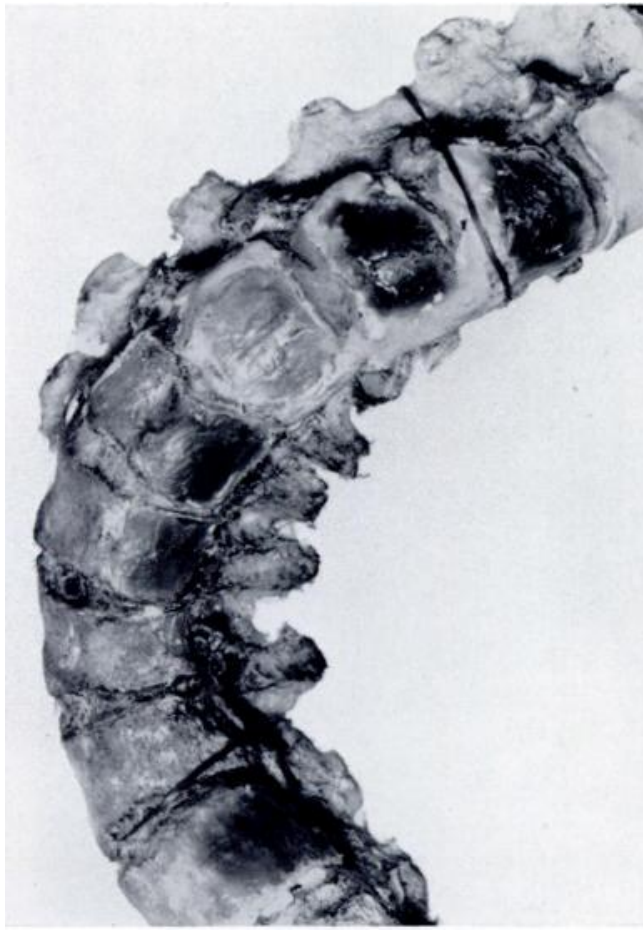

FIG. 15

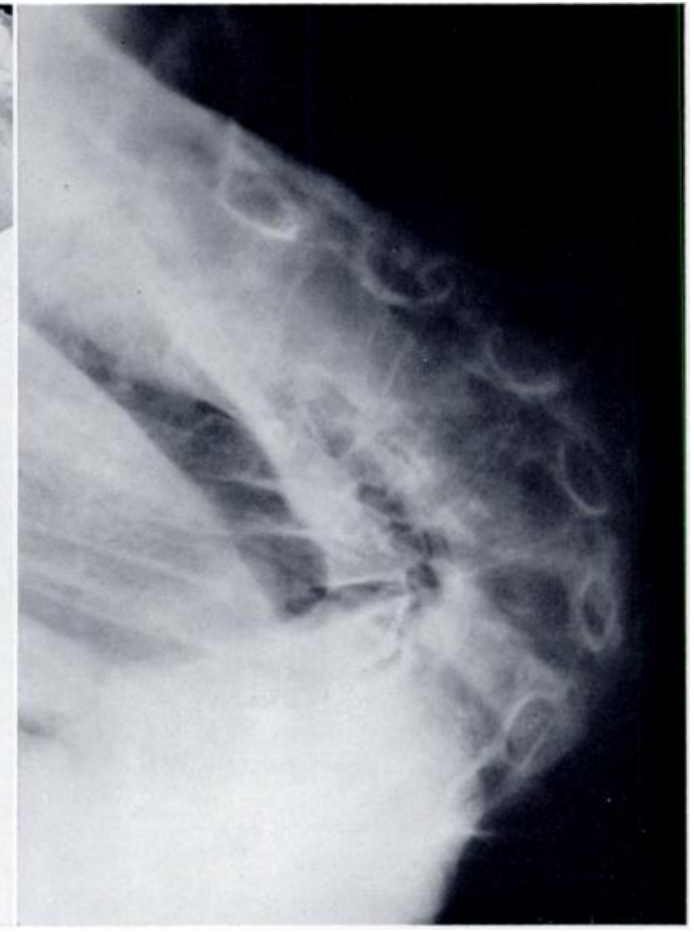

Fig. 16

Figure 15-A photograph of the apex of a curve showing that there is true lateral flexion. The vertebrae and discs are deeper on the convex side. Figure 16-A lateral radiograph of a scoliotic spine. Note that this is not a kyphosis: there is true lateral flexion in this patient. At operation to relieve paraplegia the anterior elements in this rotation were found to be longer than the posterior elements.

VOL. 48 B, NO. 4, NOVEMBER 1966 


\section{DISCUSSION}

If this concept of relative overgrowth of the anterior portion of the spine is accepted then it will be seen that under such circumstances either there must be a grotesque lordosis (which is just possible in the lumbar spine but not in the thoracic) or the longer portion must deviate sideways (Fig. 9).

The tightness of the anterior wall of the trunk-the sternum and abdominal musclesnormally prevents a very severe total lordosis. If the total length of the trunk remains short in relation to the anterior longitudinal ligament the anterior part of the spine must deviate sideways and must deviate more than the posterior part (Figs. 10 to 12). In other words torsion occurs and the greater the difference between the anterior and posterior components the greater must be the torsion. Torsion of the scoliotic spine is not necessarily related to normal movements: indeed, scoliotic torsion frequently occurs in parts of the spine where rotation does not normally occur.

Torsion is largely confined to the transitional parts of the curve (Figs. 13 and 14). At the apex of the curve there is true lateral flexion in which the depths of the convex sides of bodies, discs, pedicles (Fig. 15), intervertebral foramina and laminae are greater than the depths of the same structures on the concave side. In a typical scoliosis with rotation there is no true kyphosis but, because the central portion of the curve is already rotated, the prominent rib angles look, at first sight, like a projection of the spinous processes or a gibbus (Fig. 16). Above and below this central lateral flexion area, namely in the transition area, there is almost pure torsion with slight lordosis but little or no lateral flexion.

Anatomical study of mature specimens does not necessarily tell us what starts the pathological process, but we do know that, once a scoliosis has become established, various secondary factors, such as gravity, altered muscle action and respiration, tend to increase it and increase the relative disparity between the anterior and posterior components.

Whatever the initiating cause, disturbance in vertebral growth ultimately occurs and this takes the form of relative overgrowth of the anterior elements and is the usual continuing cause of progressive scoliosis which leads to fixed deformity.

\section{SUMMARY}

1. Evidence is presented that the basic lesion in scoliosis is relative lengthening of the anterior components of the spine compared with the posterior elements.

2. The logical treatment is to reduce this relative lengthening either by lengthening the posterior elements or shortening the anterior elements. This may be achieved by anterior lumbar wedge osteotomy or by epiphysiodesis; and correction of lumbar lordosis can improve a thoracic scoliosis.

\section{REFERENCES}

Chaklin, V. D. (1958): The Pathology, Clinical Features and Treatment of Scoliosis. Ortopedia, Traumatologia and Protezirovanie, 4, 3.

Meyer, G. H. (1866): Die Mechanik der Skoliose. Virchows Archiv für pathologische Anatomie und Physiologie, 35, 225.

Movshovich, E. A. (1960): The Question of the Pathogenesis of Scoliosis. Ortopedia, Traumatologia and Protezirovanie, No. 5, 7, 23.

Movshovich, E. A. (1963): The Surgical Anatomy of the Lumbar Portion of the Spine in Scoliosis. Ortopedia, Traumatologia and Protezirovanie, 4, 3.

Nicolodani, C. (1909): Anatomie und Mechanismus der Skoliose. Berlin and Wien: Urban \& Schwarzenberg.

Roaf, R. (1963): The Treatment of Progressive Scoliosis by Unilateral Growth-arrest. Journal of Bone and Joint Surgery, 45-B, 637. 